Technical Report No. 32-796

\title{
Electron Density and Temperature Measurements in the Exhaust of a MPD Source
}

\author{
A. J. Kelly \\ N. M. Nerheim \\ J. A. Gardner
}
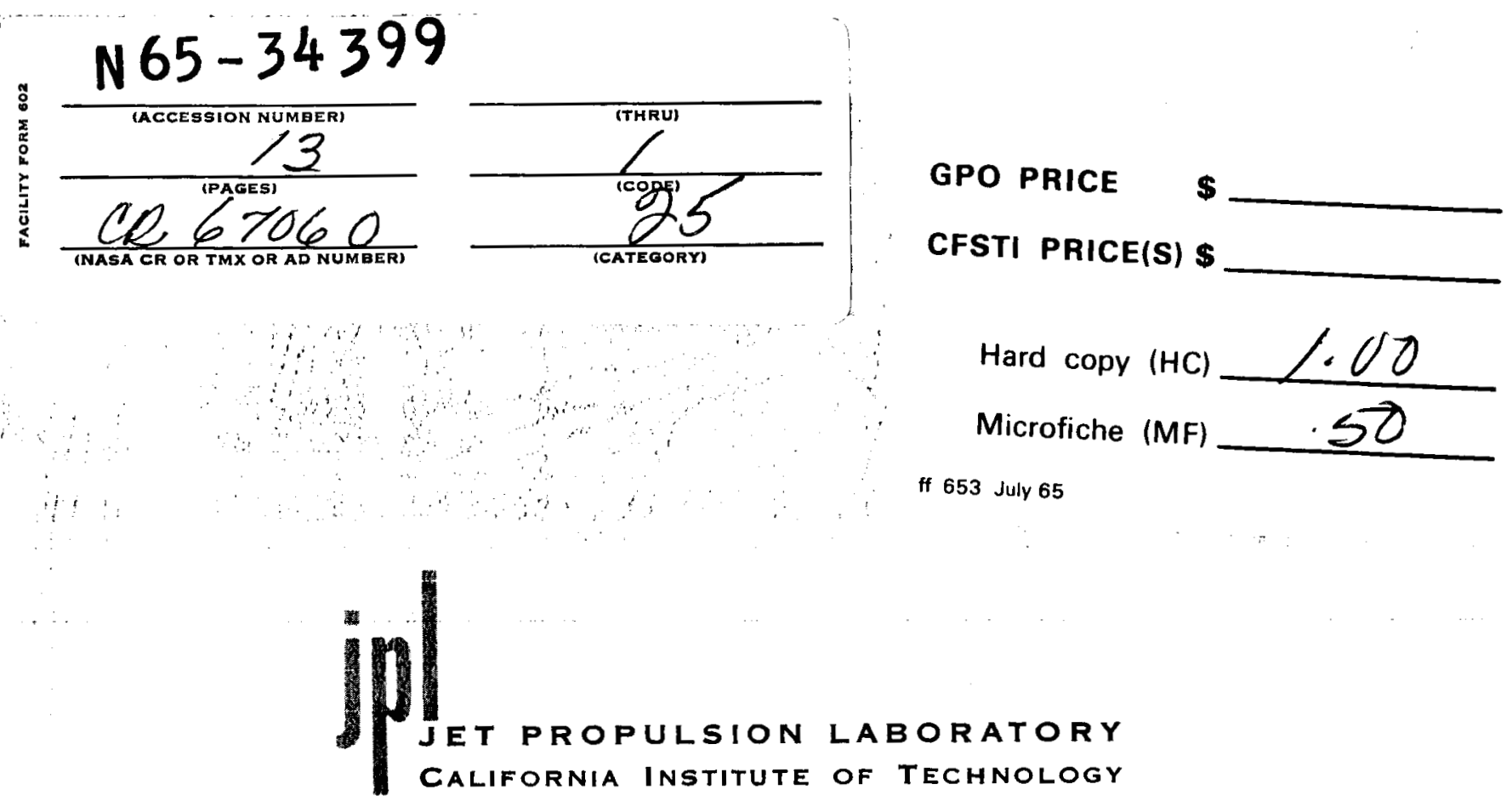

Pasadena. California 
NATIONAL AERONAUTICS AND SPACE ADMINISTRATION

Technical Report No. 32-796

Electron Density and Temperature Measurements in the Exhaust of a MPD Source

\author{
A. J. Kelly
}

N. M. Nerheim

J. A. Gardner

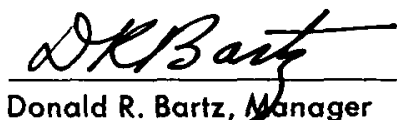

Donald R. Bartz, Manager

Propulsion Research and Advanced

Concepts Section

\author{
JET PROPULSION LABORATORY \\ CALIFORNIA INSTITUTE OF TECHNOLOGY \\ Pasadena, California
}

September 15, 1965 
Copyright (C) 1965

Jet Propulsion Laboratory

California Institute of Technology

Prepared Under Contract No. NAS 7-100

National Aeronautics \& Space Administration 


\section{CONTENTS}

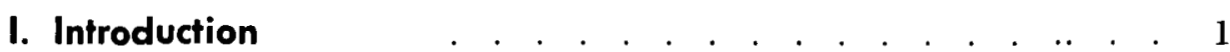

II. Description of the MPD Source . . . . . . . . . . . . . . 2

III. Diagnostic Techniques . . . . . . . . . . . . . . . . . 4

A. Langmuir Probe . . . . . . . . . . . . . . . . . . . 4

B. Microwave Diagnostics . . . . . . . . . . . . . . . . 5

C. Spectroscopic Measurements . . . . . . . . . . . . . . . . . 6

IV. Results . . . . . . . . . . . . . . . . . . . . . . . . . 6

V. Conclusions . . . . . . . . . . . . . . . . . . . . . 8

References . . . . . . . . . . . . . . . . . . . . . . . 9

\section{FIGURES}

1. Schematic diagram of Magneto-Plasma-Dynamic (MPD) source . . . 2

2. Regime of operation using argon . . . . . . . . . . . . . . . . 2

3. MPD source in operation, microwave swinging arm in background . . . 3

4. Schematic of Langmuir probe . . . . . . . . . . . . . . . 4

5. Determination of electron temperafure from Langmuir probe characteristic . . . . . . . . . . . . . . . . . . . 5

6. Electron temperature determined from the relative intensities of argon II lines . . . . . . . . . . . . . . . . . . . . . 6

7. Electron temperature along centerline of MPD plume . . . . . . . . 7

8. Electron density along centerline of MPD plume (Langmuir probe) . . . 7

9. Electron density profile at $56 \mathrm{~cm}$ downstream of arc . . . . . . . 7

10. Electron temperature profile at $56 \mathrm{~cm}$ downstream of arc . . . . . . . 7

11. Comparison of normalized electron density profiles determined by microwave and Langmuir probe methods 


\begin{abstract}
34399

Electron density and temperature profiles were measured in the exhaust of a Magneto-Plasma-Dynamic (MPD) source, operated in a vacuum facility which provides background pressures of from $\sim 100$ to $\sim 1,000 \mu$. Electron density profile measurements, made at several stations downstream of the source by using a Langmuir probe (flashed prior to each measurement) and a $24-\mathrm{Gc} / \mathrm{sec}$ microwave swinging arm probe, show agreement to within a factor of two over their common range of measurement $\left(2 \times 10^{12} \mathrm{~cm}^{-3} \rightarrow 7 \times 10^{12} \mathrm{~cm}^{-3}\right)$. Spectroscopic measurements, utilizing relative ion line intensities at the exit plane of the source, yield electron temperatures in the range 16,000 to $20,000^{\circ} \mathrm{K}$ for argon mass flows of 0.2 to $0.5 \mathrm{~g} / \mathrm{sec}$ and currents of 750 to $2,000 \mathrm{amp}$. Electron temperature profiles, measured $56 \mathrm{~cm}$ downstream of the source, have maximums of 6,000 to $7,000^{\circ} \mathrm{K}$, which are consistent with collision frequency data obtained with the microwave probe at this station. It is concluded that the plasma generated by the MPD source is in a highly nonequilibrium state. The degree of ionization at the exit of the source is lower than the equilibrium value based on the measured electron temperature. In the fully developed plume, electron temperature and density measurements show that the degree of ionization is higher than equilibrium.

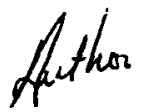

\title{
I. INTRODUCTION
}

During the past $2 \mathrm{yr}$, there has been considerable interest in the Magneto-Plasma-Dynamic (MPD) source, first described by Ducati, Gianni, and Muehlberger (Ref. 1). Thrust measurements (Refs. 2 and 3) have demonstrated that this source, because of the high specific impulse levels attained, could have significant potential as a propulsion device. These thrust measurements have indicated that at least three, and possibly more, mechanisms, both gas dynamic and electromagnetic in origin, contribute to the overall thrust level. In an effort to gain a firmer understanding of the underlying acceleration processes occurring in the device, an experimental study was undertaken of the characteristics of the exhaust plume of one such source. 
In particular, emphasis was applied to the development and application of diagnostic techniques suitable for measuring the salient characteristics of the steady plasma plume produced by the MPD source. Specifically, electron density $\left(n_{e}\right)$ measurements were made using Langmuir probe and microwave techniques. Electron temperature
$\left(T_{e}\right)$ data were obtained both spectroscopically and by the use of Langmuir probes. In addition, a limited amount of impact pressure and velocity data was collected. These data are sufficient to permit an evaluation of the degree of equilibrium and pertinent aerodynamic characteristics of the plume.

\section{DESCRIPTION OF THE MPD SOURCE}

A schematic diagram of the MPD source employed in this study is shown in Fig. 1. This source consists of a central, cylindrical, thoriated tungsten cathode, and a concentric, water-cooled copper anode. It was possible, using argon as a working fluid, to maintain stable operation at any point within the shaded region shown on the arc current $(I)$ versus mass flow $(\dot{m})$ plot of Fig. 2 . A bank of rectifiers capable of supplying up to $1 \mathrm{Mw}$ of power, with peak-to-peak ripple of $\sim 3 \%$, was used as a power supply. The MPD source operating voltage ranged from approximately 17 to $25 \mathrm{v}$. A 2.1 -m-diameter by $4.2-\mathrm{m}$-long vacuum tank exhausted by a $5,000-\mathrm{ft}^{3} / \mathrm{min}$ capacity pumping system provided a suitable environment for the operation of this source. The tank pressures attained by this pumping system were a function of the flow rate through the source. At $0.1 \mathrm{~g} / \mathrm{sec}$, the tank pressure $\left(P_{t}\right)$ was $\sim 85 \mu$, while at $0.5 \mathrm{~g} / \mathrm{sec}, P_{t} \simeq 250 \mu$; the ultimate, no-flow tank pressure was $\sim 10 \mu$.

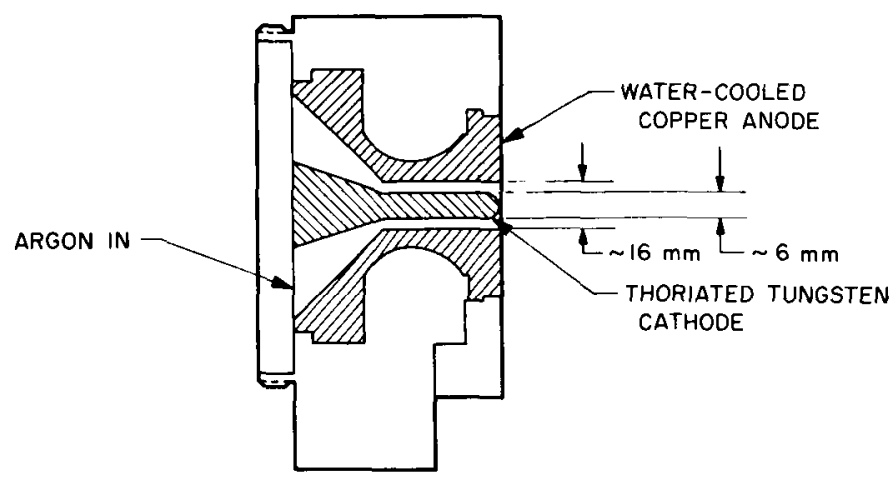

Fig. 1. Schematic diagram of Magneto-Plasma-Dynamic (MPD) source
A photo of the plume produced by the MPD source, while operating at 1,600 amp and $0.5 \mathrm{~g} / \mathrm{sec}$ of argon, is shown in Fig. 3. The jet immediately downstream of the exit of the MPD source has been found, from impact probe measurements, to be surrounded by a barrel shock, with the end of the barrel closed by a normal shock. This shock is coincident with the outer edge of the dark, globular, shell-like region which is seen to extend for some $15 \mathrm{~cm}$ downstream from the exit plane of the source. As the flow rate is increased, the normal shock pattern becomes more pronounced. Conversely, as the pressure is reduced, the entire pattern expands, and the boundaries between the various regions become less distinct. At flow rates below $0.2 \mathrm{~g} / \mathrm{sec}$, the shock pattern is not visually discernible.

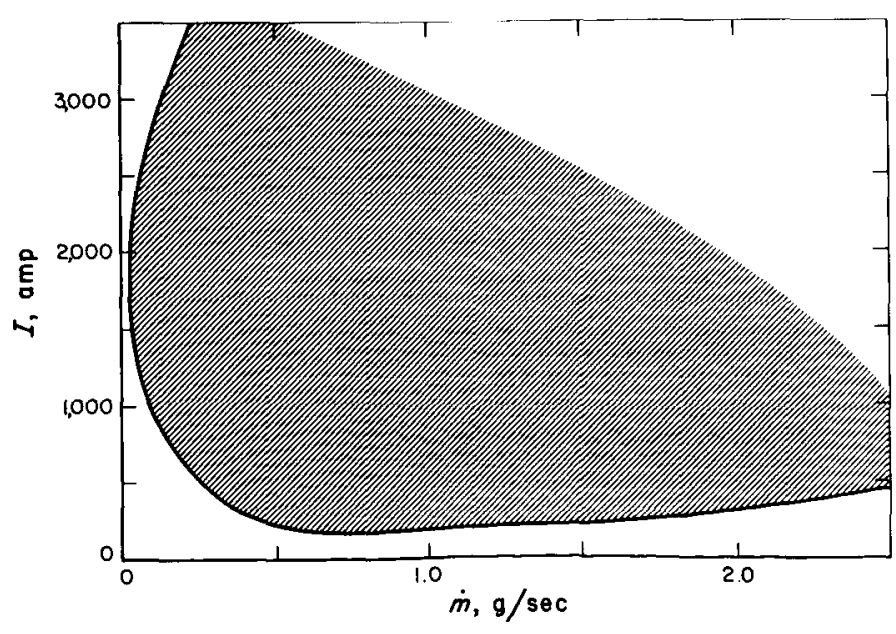

Fig. 2. Regime of operation using argon 


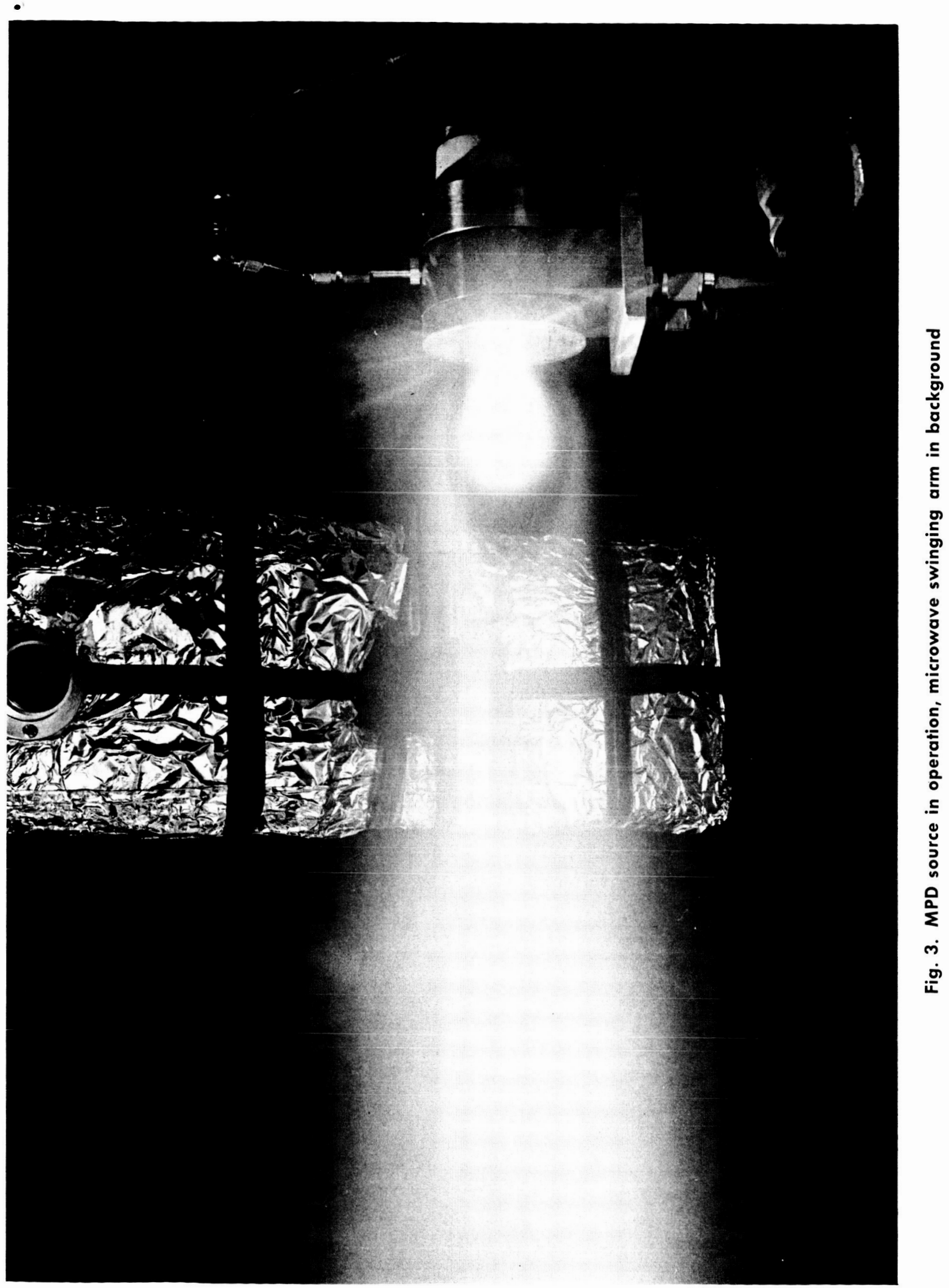




\section{DIAGNOSTIC TECHNIQUES}

\section{A. Langmuir Probe}

The Langmuir probes consisted of 1-mm-diameter by 8-mm-long tungsten pins that were insulated with 4-mmdiameter boron nitride sleeves. Two probes, aligned with the flow, were mounted $2.5 \mathrm{~cm}$ apart on a water-cooled support which could be moved both parallel and perpendicular to the plume axis. The probe bias voltage, supplied by batteries connected across a variable resistor, was varied manually from about $-40 \mathrm{v}$ to $+4 \mathrm{v}$ relative to the plasma potential. The current was plotted as a function of voltage on an $x-y$ recorder. Because of the large difference between the magnitude of the saturated ion current and saturated electron current, it was necessary to take at least three traces at different recorder sensitivities to obtain the entire probe characteristics with the required accuracy. The ion density was calculated, as suggested by Bohm (Ref. 4), from

$$
J_{i_{s a t}}=0.61 e n_{e}\left(\frac{k T_{e}}{m_{i}}\right)^{1 / 2}
$$

where $e$ is the electron charge, $k$ is Boltzmann's constant, and $m_{i}$ is the ion mass. The value of the saturated ion current density $\left(J_{i_{8 a t}}\right)$ was obtained by extrapolating the saturated ion current curve to the approximate plasma potential. The value of the electron temperature was determined by the conventional method of plotting the $\log$ of the electron current as a function of probe voltage and relating the slope to $T_{e}$. Saturated electron currents $\left(J_{e_{a t}}\right)$ could not be collected at the higher pressures because the probes became excessively hot from the large electron currents. At the lower pressures, however, both the saturated ion and electron currents were obtained. When both saturated currents were collected, the electron density was determined from

$$
J_{e_{x a t}}=\frac{e n_{e}}{4}\left(\frac{8 k T_{e}}{\pi m_{e}}\right)^{3 / 2}
$$

where $m_{e}$ is the electron mass. The current density used in the calculation was obtained by extrapolating the saturated electron current to the plasma potential. The ratio of $J_{e_{s a t}} / J_{i_{s a t}}$ was between 170 and 190 . This is in good agreement with the predicted value of 178 given by the ratio of Eq. (2) to (1). Because the frontal area of the probes consisted of less than $3 \%$ of the total probe area, the amount of additional current collected as a result of the plasma motion was assumed to be negligible.
In preliminary studies, electron densities determined by the probe were higher than those determined by the microwave measurements; the difference increased as the measurements were made closer to the MPD source. The electron density determined from the probe measurement was larger than that measured with the microwaves by a factor of two at $100 \mathrm{~cm}$ downstream of the source and by an order of magnitude at $25 \mathrm{~cm}$ downstream. To test the possibility that, when hot, the tip of the boron nitride sleeve acted as an additional collector, the saturated ion current was collected with probes of two different collecting areas. The collecting area of one probe, the test probe, was made variable by inserting a short, removable section of insulator in the boron nitride sleeve, as shown in Fig. 4. If the processes that occur at the point where the probe protrudes from the insulator remain constant when the probe area is increased by removing the section of insulator and sliding back the forward sleeve, the increased current will be due to the increased area. Rather than try to reproduce the plasma conditions exactly for runs with the two probe areas, the second lower probe was used as a monitoring probe. Many runs were conducted over a range of operating conditions and positions, and the saturated ion current was obtained from both the monitor probe and the test probe with the section of insulator in place as shown in the figure. The ratio of the currents drawn by the two probes was found to be constant within approximately $5 \%$. The section was then removed and the measurements repeated. The portion of the total current from the test probe collected by the added area was found by subtracting, from the total current, an amount equal to the product of the current

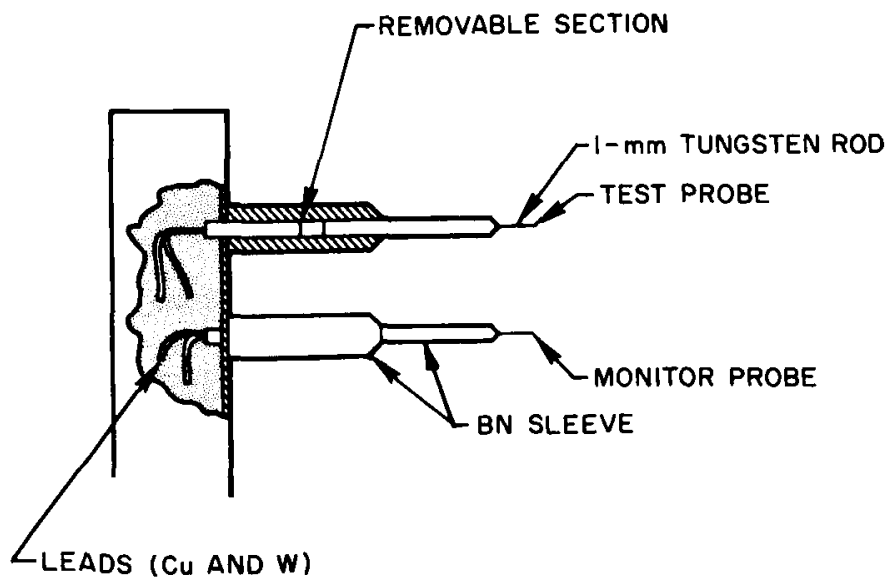

Fig. 4. Schematic of Langmuir probe 
drawn by the monitor probe and the previously determined constant. While the electron density calculated from the current collected by the added area remained nearly a factor of two larger than the value determined from the microwave measurements at $100 \mathrm{~cm}$ downstream, the anomalous effect described previously was eliminated. Probe measurements were not made at distances less than $25 \mathrm{~cm}$ from the source because the probe tip became hot and emitted electrons.

A typical curve of $\log I_{e}$, where $I_{e}$ is the electron current collected by the probe, versus the probe potential $(\Phi)$ is shown in Fig. 5. The plasma potential and the saturated electron current were obtained from the intersection of the straight lines drawn through the two linear parts of the curve. The linear relation of the curve at voltages more negative than the plasma potential indicates that the electrons possess a Maxwellian energy distribution.

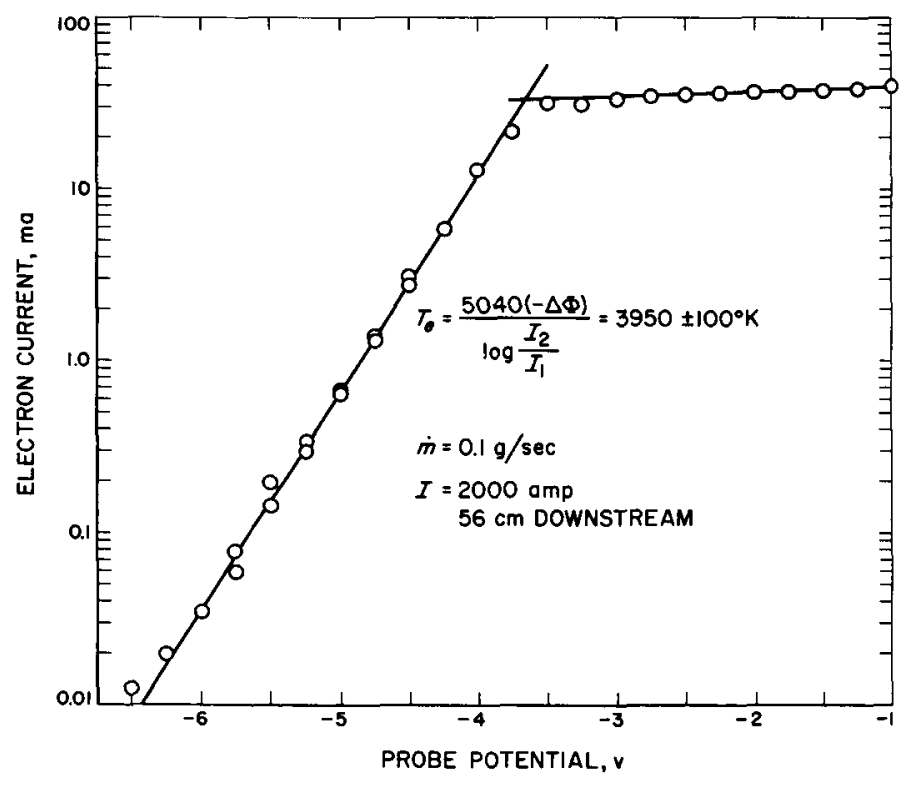

Fig. 5. Determination of electron temperature from Langmuir probe characteristic

\section{B. Microwave Diagnostics}

The swinging arm microwave probe (Ref. 5) was developed for the purpose of providing a means for measuring transverse electron density profiles of steady plasma plumes. In operation, the arm is initially latched to one side of the vacuum tank. Upon command, the latching solenoid is actuated and the arm, pivoted at a point near the top of the tank, swings in a plane, normal to the axis of the jet, and is automatically latched on the other side of the tank. The microwave transmitting and receiving horns, rigidly fixed to the arm, have matched quartz cover plates over their apertures. These plates, separated by a fixed distance, have sharp leading edges, and are positioned so that, as the arm swings, the gap formed by the plates passes over the centerline of the tank and the plume. Therefore, as the arm traverses the plasma, the microwave beam between the transmitting and receiving horns interacts with the relatively uniform, shock-free plasma slab, delineated by the quartz plates. K-band $(24 \mathrm{Gc} / \mathrm{sec})$ microwave circuitry was employed in the arm (Fig. 3). Using a gap width of $4 \lambda_{0}$ (where $\lambda_{0}$ is the free space wavelength, equal to $1.25 \mathrm{~cm}$ at $24 \mathrm{Gc} / \mathrm{sec}$ ), it was possible to attain a spatial resolution of $5 \mathrm{~cm}$ (gap width) by approximately $4 \mathrm{~cm}$ transverse to the gap and about $6 \mathrm{~cm}$ in the dimension parallel to the plume axis. Microwave energy, generated external to the vacuum tank, enters through a vacuum seal and is ducted into the circuitry on the arm via a rotating joint. The amplitude of the transmitted and reflected microwave crystal signals was measured by microwave crystal detectors mounted on the arm. The outputs from these crystals, recorded as a function of the angular position of the arm, were interpreted in terms of electron density $\left(n_{e}\right)$ and effective electron collision frequency $\left(v_{c}\right)$ using the idealized theory of a plane, plane-polarized wave interacting with a plane slab of isotropic, homogeneous plasma (Ref. 6). Corrections for the influence of near-field effects of the horns and for vagaries of the microwave crystals have been developed ${ }^{1}$ and were applied to the data.

A simple experiment was conducted in order to determine whether the presence of the microwave arm affected the electron-density profile of the plume and whether the quartz plates covering the horn apertures were significantly altering the ambient plume electron density. This experiment consisted of mounting a saturated ion probe so that it was positioned within the horn gap for one swing of the microwave arm. As soon as the arm latched upon completion of the swing, the probe, mounted on a light tubular arm and hinged on the same axis as the microwave arm, was free to retrace its path uninfluenced by the presence of the microwave arm. The two saturated ion profiles measured by this technique (one with and the other without the influence of the microwave arm) showed agreement to within approximately $20 \%$. This is consistent with the estimated total uncertainty associated

\footnotetext{
"Atom-Atom Mechanisms and Cross Sections in Noble Gases and Noble Gas Mixtures," Ph.D. thesis by A. J. Kelly, California Institute of Technology, Pasadena, California, 1965.
} 
with the interpretation of the microwave data in terms of electron density.

\section{Spectroscopic Measurements}

Spectroscopic measurements of the intensities of 12 argon II lines, made with a Jarrel-Ash 0.5-m Ebert monochromator and a 7265 photomultiplier tube, were used to determine the electron temperature by the Relative Line Intensity Method (Ref. 7). The transition probabilities given by Olsen (Ref. 8) were used in the calculation. The spectroscopic measurements were made within $5 \mathrm{~cm}$ of the arc exit because the light intensity was too low (i.e., the signal-to-noise ratio was too low) at positions further downstream to yield accurate results. A typical plot of $I_{L} \lambda / A g$ versus energy level $E$ is shown in Fig. 6; in this figure $I_{L}$ is the line intensity, $\lambda$ the wavelength of the line, $A$ the transition probability, and $g$ the statistical weight.

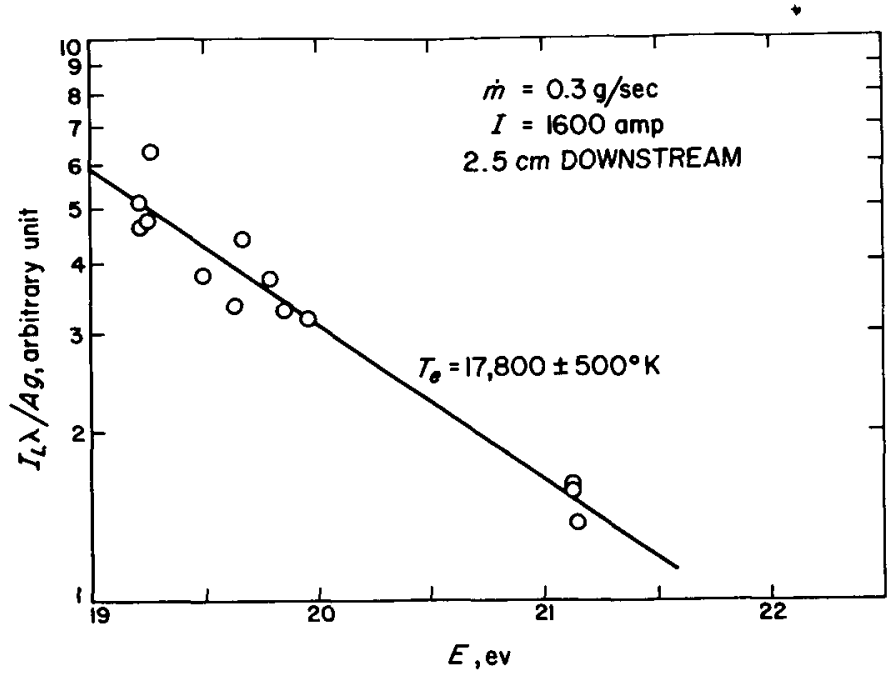

Fig. 6. Electron temperature determined from the relative intensities of argon II lines

\section{RESULTS}

Axial profiles of $T_{c}$ and $n_{e}$ along the plume centerline are shown in Figs. 7 and 8, respectively. A measure of the degree of equilibrium may be obtained by comparing the measured electron density with the equilibrium value calculated from the measured electron temperature at each position. At $100 \mathrm{~cm}$ downstream, the measured electron density is from 2 to 3 orders of magnitude higher than the calculated equilibrium value. At approximately $25 \mathrm{~cm}$, the calculated and measured densities are nearly equal. Although a similar comparison cannot be made at the arc exit because electron densities were not measured there, an estimate of the degree of equilibrium may be made from energy considerations. The spectroscopically determined temperature at the source exit was $18,000 \pm 1,000^{\circ} \mathrm{K}$. Because of the shock formation at the exit, the static pressure at the point where the temperature was measured is not known; however, with the arc operating at a flow rate of $0.3 \mathrm{~g} / \mathrm{sec}$ and a current of $1,600 \mathrm{amp}$, the anode exit pressure, measured at a wall tap approximately $2 \mathrm{~mm}$ from the exit was $19 \mathrm{~mm} \mathrm{Hg}$. An argon plasma, in which the electron density is in equilibrium with an electron temperature of $18,000^{\circ} \mathrm{K}$ and a pressure of $19 \mathrm{~mm} \mathrm{Hg}$, will contain some multiplyionized species. If the plasma were $100 \%$ singly ionized, the amount of energy tied up in ionization energy alone would be over $16,000 \mathrm{Btu} / \mathrm{lb}$. Because the input energy at the operating condition cited was less than 11,000 $\mathrm{Btu} / \mathrm{lb}$, it is apparent that the electron density must be much lower than that required for equilibrium with the measured electron temperature.

Based on these observations, it appears that a relatively small number of electrons is heated to a very high temperature in the electromagnetic field of the MPD arc, and that the ionization rate is too slow to produce equilibrium ionization levels during the period of time in which the gas is accelerated. In the field-free region, the electron temperature decays very rapidly and, near the normal shock region, the electron density is equal to the value which would correspond to equilibrium at the electron temperature. Further downstream, the electron temperature continues to decay, but the electron recombination rate is too slow to maintain the electron density in equilibrium with the electron temperature. 


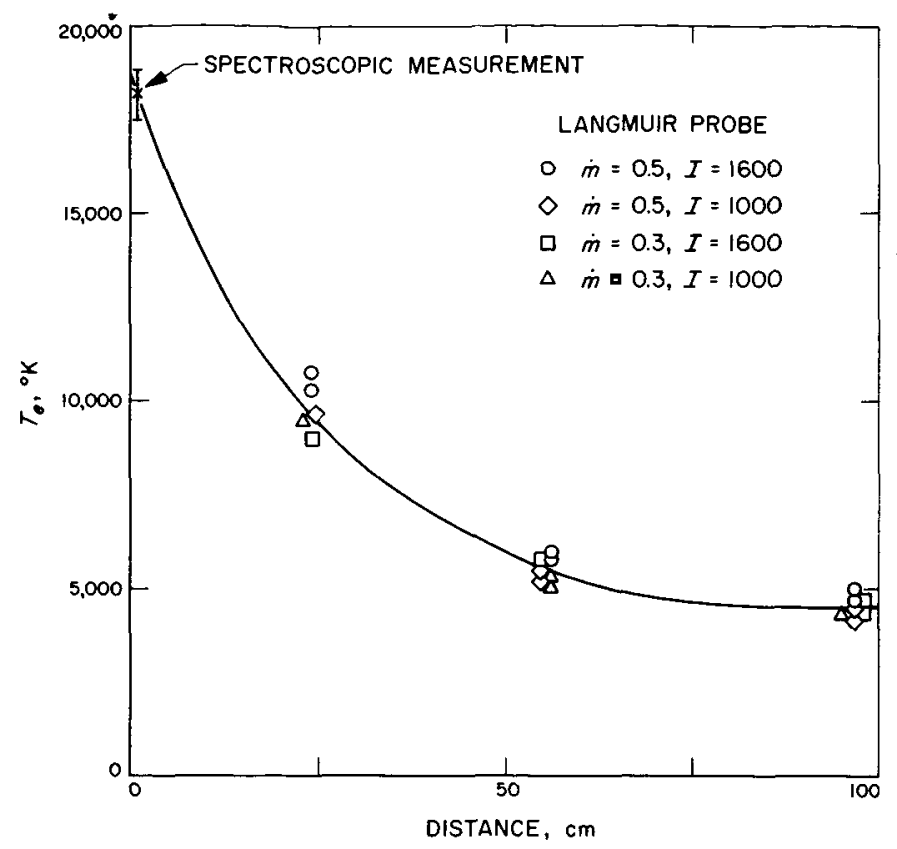

Fig. 7. Electron temperatures along centerline of MPD plume

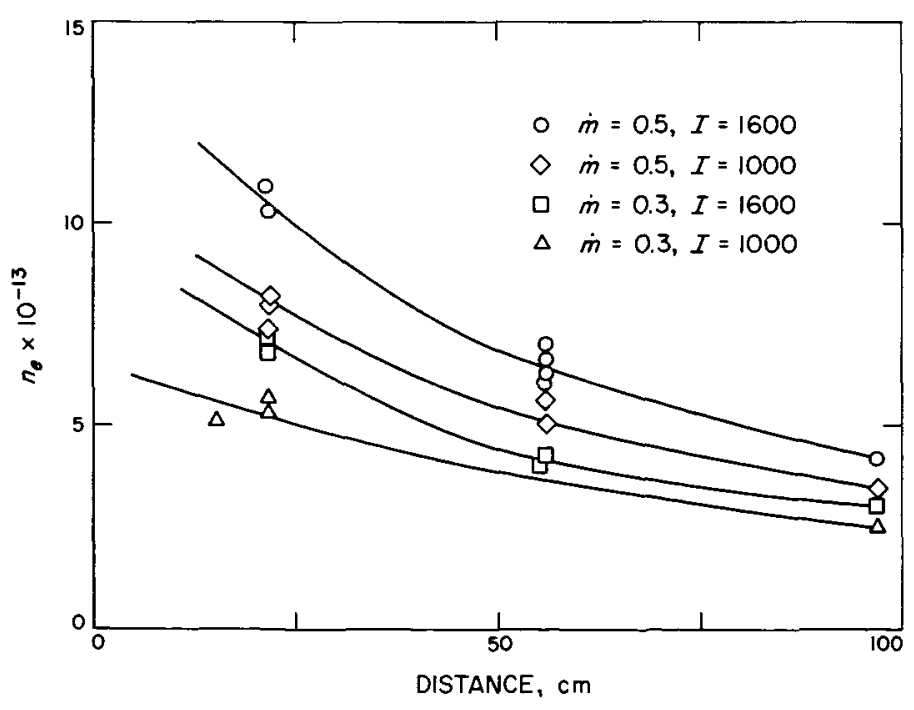

Fig. 8. Electron density along centerline of MPD plume (Langmuir probe)

Using Langmuir probe techniques, electron density and electron temperature radial profiles at a station 56 $\mathrm{cm}$ from the exit plane of the source were obtained under conditions where $I=1,600 \mathrm{amp}, \dot{m}=0.5 \mathrm{~g} / \mathrm{sec}$. These profiles are shown in Figs. 9 and 10, respectively. An electron density profile, taken under the same operating conditions and at the same longitudinal position using the microwave probe, shows agreement to within

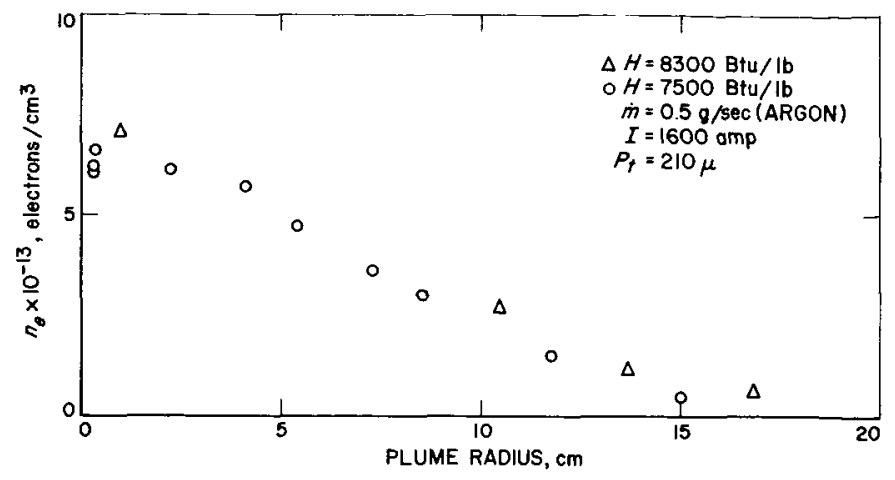

Fig. 9. Electron density profile at $56 \mathrm{~cm}$ downstream of arc

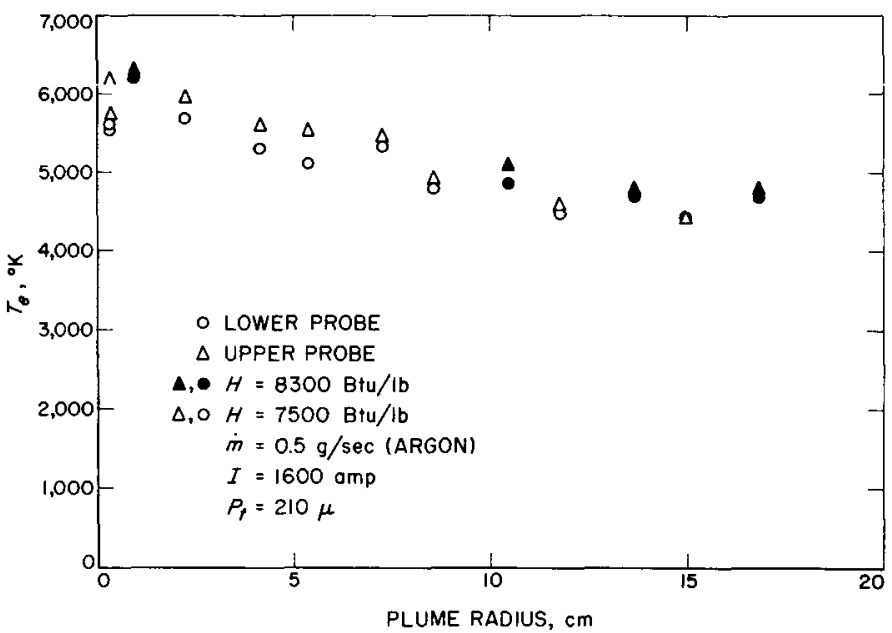

Fig. 10. Electron temperature profile at $56 \mathrm{~cm}$ downstream of are

a factor of two with the data of Fig. 9 over the range of electron density common to both techniques, i.e., $2 \times 10^{12} \leq n_{e} \leq 7 \times 10^{12} \mathrm{~cm}^{-3}$. The cut-off electron density for the 24-Gc/sec microwave probe is $\sim 7 \times 10^{12} \mathrm{~cm}^{-3}$. Therefore, the comparison could only be made on the "wings" of the profile. In addition to the determination of electron density, the microwave data permitted the Maxwell averaged electron collision frequency to be measured. Electron temperatures inferred from these data agreed to within the experimented error $\left( \pm 1,000^{\circ} \mathrm{K}\right)$ with those obtained with the Langmuir probe.

In order to effect a more complete comparison of the microwave and Langmuir probe data, the MPD source was operated at conditions where the centerline electron 
density $n_{e, \notin}$ was below the cut-off level of the microwave probe. Electron density profiles, determined by both techniques at a position $56 \mathrm{~cm}$ from the source, are shown in Fig. 11. The electron density data are normalized to the conditions at the centerline $\left(n_{e . q}\right)$ which, in the case of the microwave data, were $5.0 \times 10^{12} \mathrm{~cm}^{-3}$. The normalization factors, i.e., $n_{e, \notin}$, were found to differ by as much as a factor of two. The profiles are seen to be quantitatively equivalent. Comparison of the profile data taken at $0.1 \mathrm{~g} / \mathrm{sec}$ with that taken at $0.5 \mathrm{~g} / \mathrm{sec}$ (Fig. 9) reveals that the electron density profile is much flatter at the lower flow rate.

The impact pressure downstream of the normal shock indicated a Mach number of about unity. A direct measurement of the flow velocity was made by measuring the potential difference between two points in the exhaust jet along a line perpendicular to both the flow direction and an applied magnetic field (Ref. 9). The measurements were made by varying the current to the magnet and relating the slope of the resulting potential versus cur-

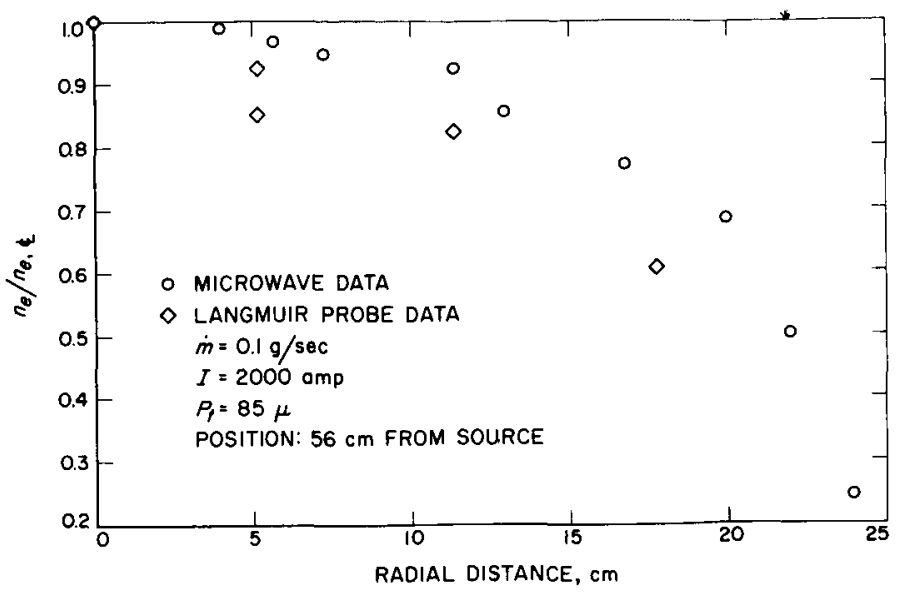

Fig. 11. Comparison of normalized electron density profiles determined by microwave and Langmuir probe methods

rent curve to the velocity. Downstream of the normal shock, the velocity was found to be about $10^{5} \mathrm{~cm} / \mathrm{sec}$, and also indicated a Mach number of about unity.

\section{v. CONCLUSIONS}

1. Gas enthalpy calculated from power input, cooling water losses, and gas flow measurements show that, at the exit of the source, the degree of ionization must be much lower than equilibrium based on the spectroscopically determined electron temperature.

2. Measured electron density levels in the plume downstream of the shock region are much larger than electron densities calculated from the Saha equation employing measured electron temperatures.

3. Langmuir probe measurements indicate that, in the plume, the electron energy distribution is Maxwellian.

4. Electron density measurements made with the Langmuir and microwave probes show agreement to within a factor of two.

5. Immediately downstream of the source, impact probe measurements revealed the existence of a barrel shock closed by a normal shock. Downstream of this shock, impact and velocity probe measurements indicate that the Mach number of the plume is close to unity. 


\section{REFERENCES}

1. Ducati, A. C., Gianni, G. M., and Muehlberger, E., "Experimental Results in HighSpecific-Impulse Thermo-Ionic Acceleration," Journal of the AIAA, Vol. 2, p. 1452, 1964.

2. John, R. R., Bennett, S., and Connors, J. F., "Experimental Performance of a High Specific Impuise Arc Jet Engine," AlAA Paper No. 64-669, AlAA Fourth Electric Propulsion Conference, Philadelphia, Pa., Aug. 31-Sept. 2, 1964.

3. Cann, G. L., "Annular Magnetic Hall Current Accelerator," AlAA Paper No. 64-670, AIAA Fourth Electric Propulsion Conference, Philadelphia, Pa., Aug. 31-Sept. $2,1964$.

4. Bohm, D., Burhop, E. H. S., and Massey, H. S. W., "The Characteristics of Electrical Discharges in Magnetic Fields," Eds., A. Guthrie and R. K. Wakerling, Chapter 2. McGraw-Hill, New York, 1 y 49.

5. Kelly, A. J., "A Microwave Probe for Plasma Plumes," Journal of the AIAA, Vol. 3, p. $372,1965$.

6. Jahn, R. G., "Microwave Probing of lonized-Gas Flows," The Physics of Fluids, Vol. 5, No. 6, p. 678, 1962.

7. Dieke, G. H., "Physical Measurements in Gas Dynamics and Combustion," Ed., R. W. Sadenburg, et al., Princeton University Press, p. 491, 1954.

8. Olsen, H. N., "The Electric Arc as a Light Source for Quantitative Spectroscopy," J. Quant. Spectrosc. Radiat. Transfer, Vol. 3, p. 305, Pergamon Press Ltd., 1963.

9. Russell, G. R., Byron, S., and Bortz, P. I., "Performance and Analysis of a NonEquilibrium Crossed Field Accelerator," AIAA Electric Propulsion Conference, Colorado Springs, March 1963. 